\title{
Domain Decomposition Techniques for Parallel Generation of Tetrahedral Meshes
}

\author{
Barbara Głut and Tomasz Jurczyk \\ AGH University of Science and Technology, Kraków, Poland \\ \{glut, jurczyk\}@agh.edu.pl
}

\begin{abstract}
We present solutions for dealing with the problem of parallel generation of unstructured meshes for 3D domains. The selected approach is based on a geometric decomposition of the domain where the input data is given in the form of a surface mesh. The difference between the two presented decomposition techniques lies in the step where the actual partitioning takes place. In the first method the partitioning is obtained using solely the surface mesh while in the latter one a coarse tetrahedral mesh is used. After the decomposition and creation of an interface mesh (which is a surface mesh in both approaches) the final volume meshes are generated independently for each subdomain. The interface mesh and the subdomain meshes are constructed using a Riemannian metric combined with a control space structure which allows to generate meshes with varied density and anisotropy [1].
\end{abstract}

Keywords: Mesh Generation, Geometric Decomposition, Tetrahedral Mesh, Anisotropic Metric.

\section{Introduction}

In the modern simulations of processes with the finite element method the increasingly more complicated models require very large number of elements in order to achieve sufficiently precise computations. Consequently, such computational tasks are often solved using a parallel approach. However, the parallelization of the solver does not solve the problem completely. The sequential construction of meshes with a large number of elements poses some problems as well - mainly with respect to the memory requirements. It should be noted that the problem of a parallel mesh generation is considered much more difficult than the parallelization of the further computation step 2. An efficient parallel algorithm requires an adequate load balancing for computational nodes while minimizing the communication overhead between the processors.

The task of decomposing the domain for the subsequent mesh generation is complicated due to the limited information available at the beginning of this process (which usually includes only the geometric model description). At this point it is usually difficult to properly assess the time required to discretize the subdomains of the created partitioning which is necessary to achieve the proper

M. Bubak et al. (Eds.): ICCS 2008, Part I, LNCS 5101, pp. 641 650 2008.

(C) Springer-Verlag Berlin Heidelberg 2008 
load balancing. An additional complication is often introduced by an irregular density and anisotropy of the mesh in some areas of the discretized model 1

In recent years much notice has been devoted to the problem of a parallel mesh generation and a number of solutions have been proposed [3]. It seems that the key to the successful parallelization of the mesh generation problem is the proper partitioning of the domain into subdomains and a possibly independent discretization of these subdomains. Depending on the method and the order of an interface mesh generation three classes of methods can be identified [4]:

1. The a priori class includes methods which first create meshes of the interfaces and then, in parallel, the meshes for each subdomain are generated.

2. The a posteriori methods generate in parallel the meshes of the subdomains first. These meshes are then adjusted in a way which assures consistency of the mesh within the whole domain.

3. The third class contains methods where the interface meshes and the subdomain meshes are generated concurrently.

There have been so far developed no methods which would solve this problem in a satisfactory way for a wide class of 3D models. It is partly due to the fact that even the sequential problem of the volume mesh generation for arbitrary domains is difficult enough. A number of different techniques of mesh construction is utilized in various generators which makes it difficult to choose the most advantageous class of the parallelization methods for the given task. Additionally, the geometric complexity of the considered models is constantly increasing which reduces the chances of finding a definite solution for this problem. However, due to the importance of this problem the heuristic solutions applicable in a possibly wide family of models have to be sought.

\section{Main Concept of the Proposed Techniques}

Two approaches of decomposing the discretized domain into subdomains are presented in the article. For both methods the input data are the boundary surface meshes. The difference between these methods is the selection of a moment when the parallelization procedure is executed. Both methods can be categorized as the $a$ priori class where the interface meshes are constructed first and then the mesh of each subdomain can be independently generated in parallel. Such approach has a number of advantages. During the parallel generation of the mesh the communication between the computational nodes is limited. The volume mesh does not need to be stored in the memory of a single computational node. The only data interchanged during the simulation phase is the information about interface meshes which have to be compatible. Moreover, the sequential mesh generator can be utilized without any modifications. This technique assures also keeping the initial surface mesh intact which can be beneficial for the computational process.

${ }^{1}$ Such requirements concerning the shape and density of elements may result for example from the computational aspects or the geometric features of the domain. 
The studies presented in this article are founded on the mesh generator developed by authors [1. This generator constructs meshes using the information gathered in the control space [5]. The concept of a Riemannian metric stored within the adaptive control space structure has a substantial influence on the proposed techniques of the mesh decomposition for a parallel generation. As a consequence the developed methods can be successfully used in the problems with a high local variation of the density and the anisotropy of the mesh which are often found in the contemporary simulations with the adaptive solvers.

\section{Method I: Decomposition of Surface Mesh (DSM)}

The DSM technique (Fig. 1) 8] is based on the geometric decomposition of the domain using the surface meshes only. The surface mesh is partitioned by cutting it with separators which at this development stage are implemented as planes. Then, the subdomains are being closed by generation of a proper surface mesh on the separators. Finally, the volume mesh can be constructed independently for each of these closed subdomains. The main steps for each closed subdomain:

1. Selection of the separator.

2. Localization of the intersection of the separator with the surface mesh and determination of the cutting contours.

3. Generation of a surface mesh on the separator (Fig. 1(b) which in case of a planar separator requires:

- construction of a $2 \mathrm{D}$ control space taking into account various metric sources,

- generation of a 2D mesh on the cutting plane,

- projection of the planar mesh to the $3 \mathrm{D}$ space.

4. Closing of the subdomains (Fig. 1(c)).

5. Generation of volume meshes in the subdomains (Fig. 1(d)).

The selection of a separator (with respect to both its shape and placement) is crucial for the final effect of the domain decomposition as well as for the course of the subsequent phases of the method. The selection of the separator should assure a low cut size, a proper load balancing and a minimal number of multiply connected interfaces. In the literature two main techniques are usually proposed for the selection of the cutting plane: along the inertial axis [6] and perpendicularly to the longest edge of the bounding cubicoid [7]. However, none of these methods guarantee sufficiently good results in a general case and this problem is considered as a subject of further studies. In the presented examples the authors applied cutting using the information about the bounding box.

The construction of an interface mesh requires first the localization of cutting contours of the surface mesh and the separator. These contours are then projected onto the separator plane. In order to generate the mesh of the cutting plane, a special 2D control space is created. The metric in this case is associated with the lengths of edges in the cutting contour calculated both in the threeand two-dimensional space. Any other available metric sources are also included. 


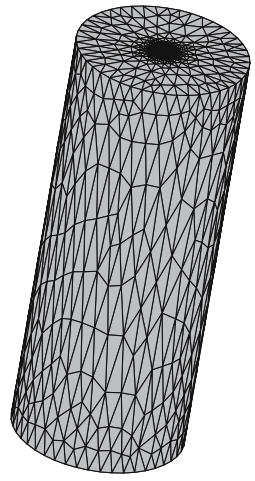

(a) surface mesh

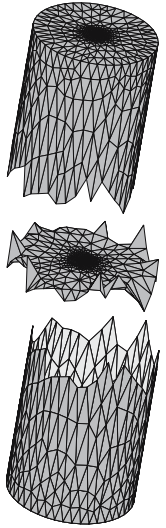

(b) split

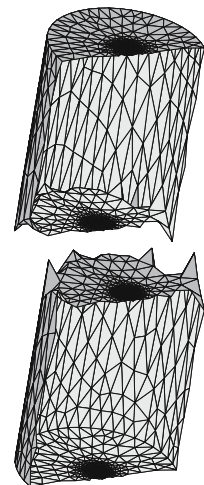

(c) closing (cross-section)

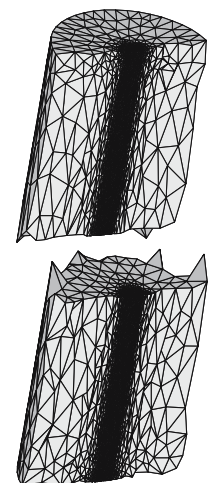

(d) final mesh (cross-section)

Fig. 1. Subsequent steps of DSM (the cross-section visualization of a mesh is created by removing a set of elements)

Using the created metric field the $2 \mathrm{D}$ mesh is generated and projected to the $3 \mathrm{D}$ space. This technique was described in more detail in 8.9 where different problems respecting the placement of a separator were also considered.

\section{Method II: Decomposition of Coarse Volume Mesh (DCVM)}

In the second method (Fig. 22) the coarse volume mesh is utilized to partition the discretization domain. This coarse mesh is created as a result of a discretization based on the boundary nodes only. In this technique the separators are purely virtual and their purpose is to guide the mesh refinement in the selected subdomain. The partitioning of the domains is achieved by separation of the mesh along the refined fragments of the mesh which also defines the boundaries of closed subdomains. The meshes for the closed subdomains are generated independently as in the first approach. In this method the cost of the sequential part of the procedure increases but a more detailed information about the discretized domain becomes available which might help to achieve a better decomposition.

The subsequent steps of this method:

1. Generation of a coarse 3D mesh (Fig. 2(a)).

2. Determination of the separator placement.

3. Refinement of the mesh in the vicinity of the separator (Fig. 2(b)) .

4. Separation of the subdomains and recognition of the interface surface (Fig. 2(c)).

5. Refinement of the volume meshes in the subdomains (Fig. 2(d)].

The coarse mesh based on the boundary nodes only is created with utilization of a three-dimensional control space. The contents of this space is determined 


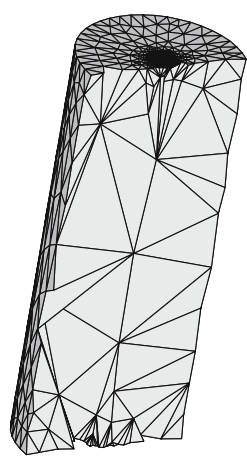

(a) coarse mesh (cross-section)

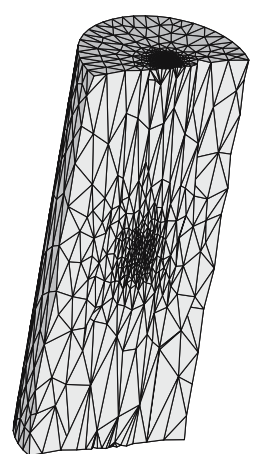

(b) refinement (cross-section)

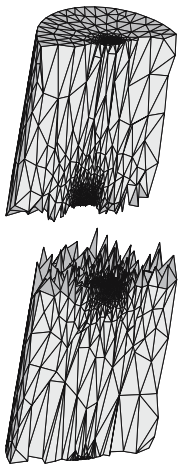

(c) split (cross-section)

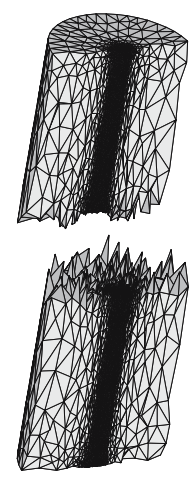

(d) final mesh (cross-section)

Fig. 2. Subsequent steps of DCVM

using the geometry of the model and any additional criteria which may be introduced by the user.

As in the DSM, the first problem which has to be solved is determining the placement of the virtual separator. The solutions proposed in the literature for methods starting decomposition from coarse mesh are usually applying partitioning libraries (like $\mathrm{METIS}^{2}$, $\mathrm{CHACO}^{3}$, etc.) [10, neural networks or genetic algorithms [1]. However, in order to better compare both presented methods the selection of a separator is determined in this work similarly as in the DSM.

In the vicinity of the separator the control space (and the metric stored therein) is modified in order to obtain a selective refinement of the mesh in the subsequent step of the procedure. This special control space $\mathrm{CS}_{p}^{3 d}$ used to prepare the coarse mesh for partitioning is calculated using the main control space $\mathrm{CS}_{m}^{3 d}$. The metric near the separator is copied directly from $\mathrm{CS}_{m}^{3 d}$ and in the other areas of the discretized domain the maximum metric is applied (with an additional smoothing of the metric field).

The mesh created with respect to $\mathrm{CS}_{p}^{3 d}$ is partitioned along the virtual separator which at this point is already properly refined. The procedure of an actual mesh partitioning starts with identification of all faces incident to two tetrahedral blocks belonging to a different partitions. In order to reduce the cut size (i.e. the number of faces between the partitions) an additional operation of moving some mesh elements between the partitions is performed. Finally, the mesh elements from different partitions are divided into the separate meshes which requires duplication of all mesh entities (vertices, edges and faces) forming the interface mesh and some updating of the mesh interconnections (all these operations are local). The volume meshes in the subdomains can be then further refined independently and the discretization of each subdomain is guided by the main control space $\mathrm{CS}_{m}^{3 d}$.

\footnotetext{
2 http://glaros.dtc.umn.edu/gkhome/views/metis/

3 http://www.cs.sandia.gov/ bahendr/chaco.html
} 


\section{Examples}

Both proposed methods were inspected for various geometric models and discretizations of theirs surfaces. The test meshes are shown in Fig. 3.

The results of the mesh generation via domain decomposition (with one separator) using both described methods are shown in Fig. 4, 5, 6, and 7. Since the article concentrates on the partitioning method itself, all test were computed on a single machine.

Table 1 presents the numbers of elements in the volume meshes created using different approaches.

For both presented methods the summary number of tetrahedra is similar as in the case of the sequential generation. The only significant difference between the methods is visible in the number of faces on the interface between the mesh partitions.

In Table 2 there are gathered the running costs (for a single $3.2 \mathrm{GHz}$ Intel P4 computer with 1 GB memory) of the subsequent steps of the mesh generation process. Both tested methods allow to decrease the expected parallel meshing time 4 The running times for the DCVM method are somewhat higher than for the DSM method. In this case the increased time is mostly due to the cost of the initial sequential step. The times of the volume mesh construction in the partitioned subdomains using the second method (DCVM) are lower since the coarse volume meshes for the subdomains are already available. Also the boundary recovery cost is absent (since this procedure had been already run in the earlier sequential part) which is most visible in the case of the mesh $M_{3}$ (a non-convex domain). The quality of meshes (Table 3) obtained using both the first and the second method is very similar and also close to the quality of the meshes generated sequentially.

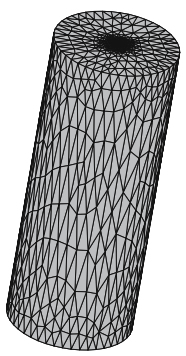

(a) $M_{1}$

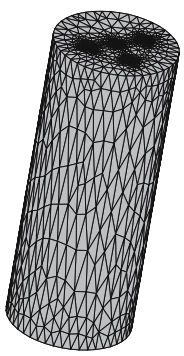

(b) $M_{2}$

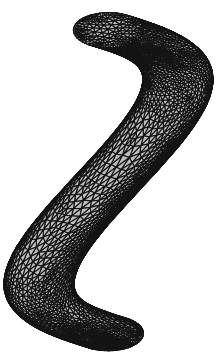

(c) $M_{3}$

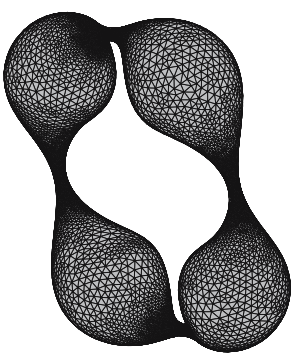

(d) $M_{4}$

Fig. 3. Example meshes

\footnotetext{
${ }^{4}$ The given summary time does not include the cost of transferring the partition data between the computation nodes which may depend on the specific parallel architecture.
} 


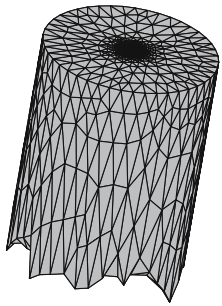

(a) DSM

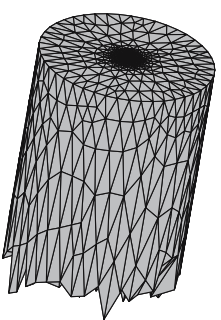

(b) DCVM

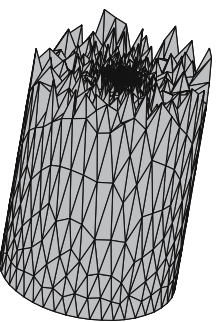

Fig. 4. Decomposition of the mesh $M_{1}$

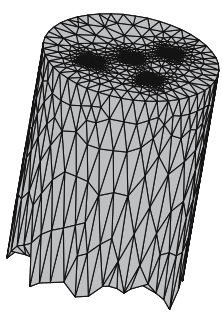

(a) DSM

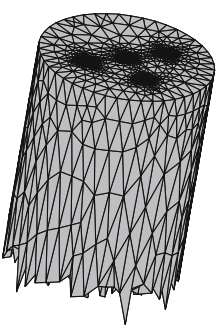

(b) DCVM

Fig. 5. Decomposition of the mesh $M_{2}$

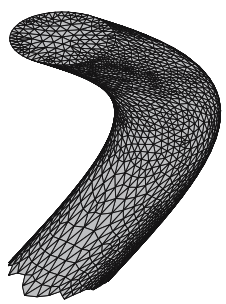

(a) DSM
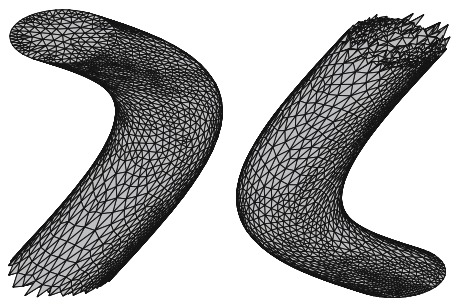

(b) DCVM

Fig. 6. Decomposition of the mesh $M_{3}$

Table 1. Number of elements $\left(\mathrm{NF}_{B}\left[10^{3}\right]\right.$ - number of boundary faces, NT $\left[10^{3}\right]-$ number of tetrahedra, $\mathrm{NT}_{i}\left[10^{3}\right]-$ number of tetrahedra in $i^{\text {th }}$ partition, $\mathrm{NF}_{I}$ - number of faces on interface (cut size))

\begin{tabular}{r|r|r|rrr|rrr|rr}
\hline \multicolumn{2}{r|}{} & Sequential & \multicolumn{4}{|c|}{ DSM } & \multicolumn{3}{c}{ DCVM } \\
\hline Mesh & $\mathrm{NF}_{B}$ & $\mathrm{NT}$ & $\mathrm{NT}_{1}$ & $\mathrm{NT}_{2}$ & $\mathrm{NT}_{(1+2)}$ & $\mathrm{NF}_{I}$ & $\mathrm{NT}_{1}$ & $\mathrm{NT}_{2}$ & $\mathrm{NT}_{(1+2)}$ & $\mathrm{NF}_{I}$ \\
\hline$M_{1}$ & 4.1 & 427.2 & 209.1 & 206.5 & 415.6 & 1566 & 214.2 & 227.4 & 441.6 & 3657 \\
$M_{2}$ & 6.5 & 794.9 & 380.9 & 377.0 & 757.9 & 2693 & 396.5 & 432.0 & 828.6 & 7152 \\
$M_{3}$ & 7.9 & 83.9 & 41.7 & 44.1 & 85.8 & 391 & 43.1 & 43.2 & 86.3 & 654 \\
$M_{4}$ & 31.1 & 254.6 & 127.2 & 119.5 & 246.7 & 432 & 133.1 & 127.7 & 260.8 & 1075 \\
\hline
\end{tabular}




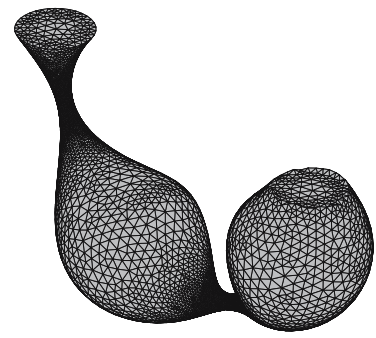

(a) DSM

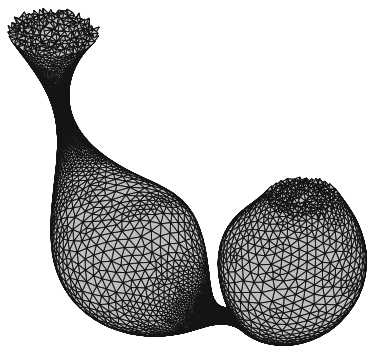

(b) DCVM

Fig. 7. Decomposition of the mesh $M_{4}$ (only one of the created subdomains is shown)

Table 2. Generation time $\left(t_{s}[s]\right.$ - sequential generation time, $t_{i}[s]$ - mesh generation time for the $i^{\text {th }}$ subdomain, the summary parallel generation time $t_{\text {sum }}$ is estimated as $\left.t_{s}+\max \left(t_{i}\right)\right)$

\begin{tabular}{|c|c|c|c|c|c|c|c|}
\hline & Sequential & \multicolumn{2}{|l|}{ DSM } & \multicolumn{4}{|c|}{ DCVM } \\
\hline Mesh & $t_{s}$ & $t_{1} \quad t_{2}$ & $t_{\text {sum }}$ & $t_{s}$ & $t_{1}$ & $t_{2}$ & $t_{\text {sum }}$ \\
\hline$M_{1}$ & 42.1 & 0.417 .116 .7 & 17.5 & 14.0 & 15.4 & 16.7 & 30.7 \\
\hline$M_{2}$ & 80.0 & 0.532 .433 .0 & 33.6 & 27.1 & 29.13 & 32.5 & 59.6 \\
\hline$M_{3}$ & 9.6 & $\begin{array}{lll}0.2 & 4.2 & 6.0\end{array}$ & 6.2 & 6.0 & 2.6 & 2.7 & 8.7 \\
\hline$M_{4}$ & 25.1 & 1.012 .011 .3 & 13.0 & 10.4 & 9.1 & 8.7 & 19.5 \\
\hline
\end{tabular}

Table 3. Quality of the generated meshes $\left(\bar{\eta}_{\mathcal{M}}\right.$ - average mean ratio of mesh elements calculated in metric space, $\eta_{\mathcal{M}}^{\min }$ - minimum mean ratio of mesh elements calculated in metric space, $\bar{\mu}_{\mathcal{M}}$ - average length of mesh edges calculated in metric space [12])

\begin{tabular}{|c|c|c|c|}
\hline & Sequential & DSM & DCVM \\
\hline Mesh & $\bar{\eta}_{\mathcal{M}} \eta_{\mathcal{M}}^{\min } \bar{\mu}_{\mathcal{M}}$ & $\bar{\eta}_{\mathcal{M}} \eta_{\mathcal{M}}^{\min } \bar{\mu}_{\mathcal{M}}$ & $\bar{\eta}_{\mathcal{M}} \eta_{\mathcal{M}}^{\min }$ \\
\hline$M_{1}$ & $0.8820 .167 \quad 1.037$ & 0.8810 .0341 .018 & 0.8820 .0691 .037 \\
\hline$M_{2}$ & $\begin{array}{llll}0.874 & 0.050 & 1.031\end{array}$ & 0.8740 .0091 .008 & 0.8740 .0251 .031 \\
\hline$M_{3}$ & 0.8580 .0351 .034 & 0.8580 .0921 .025 & 0.8460 .0011 .046 \\
\hline$M_{4}$ & 0.8750 .1091 .040 & 0.8740 .1531 .027 & 0.8750 .0651 .040 \\
\hline
\end{tabular}

\section{Conclusions}

The DSM method based on the generation of a mesh on a separator surface has the benefit of a low cost of the sequential part and a small cut size (for a given selection of a separator). However, this technique is sensitive to a proper placement of a separator [8]. If the angle between the separator and the surface mesh is too small (which can be difficult to avoid for complex models) there may arise problems with the correct projection of contour nodes onto the separator surface. Moreover, the quality of the volume elements generated in such areas may be unacceptably low. 
The second method (DCVM) overcomes this problem and the quality of the generated mesh elements is unaffected by the selection of the (virtual) separator placement. The separator type for this method can be also easily extended to a non-planar surface. Moreover, due to the availability of the coarse volume mesh during the partitioning phase the predicted number of elements in the final mesh for various areas of the discretized domain might be assessed with higher accuracy. As a result better balancing of the decomposition balancing may be achieved. Unfortunately, these benefits are combined with an increased cost of the sequential part of the algorithm and a higher cut size.

\section{Further Research Directions}

The computational and communication costs are different for various computer architectures. Because of this it is difficult to select the proper parallelization strategy applicable for different architectures. From this point of view the approach where each subdomain becomes an individual object to discretize appears to be advantageous. However, this thesis has to be tested and verified for a number of different architecture configurations and tools.

The further studies are also required with respect to the localization of the optimal placement and shape of the separator. This task is correlated with the problem of assessment of the predicted number of volume elements in the final mesh based only on the number of boundary elements. The authors were inspecting a similar problem for a two-dimensional case [13. A further studies are however necessary for three-dimensional meshes where the prediction will additionally utilize the information from the control space.

Acknowledgments. The partial support of the AGH Grant No. 11.11.120.777 is gratefully acknowledged.

\section{References}

1. Głut, B., Jurczyk, T., Kitowski, J.: Anisotropic Volume Mesh Generation Controlled by Adaptive Metric Space. In: AIP Conf. Proc. NUMIFORM 2007, Materials Processing and Design: Modeling, Simulation and Applications, Porto, Portugal, June 17-21, vol. 908, pp. 233-238 (2007)

2. Tu, T., Yu, H., Ramirez-Guzman, L., Bielak, J., Ghattas, O., Ma, K.-L., O'Hallaron, D.R.: From Mesh Generation to Scientific Visualization: An End-toEnd Approach to Parallel Supercomputing. In: Proc. of SC 2006, Tampa, FL (2006)

3. Chrisochoides, N.: A survey of parallel mesh generation methods, http://www.cs.wm.edu/ nikos/pmesh_survey.pdf

4. Cougny, H.L., Shepard, M.S.: Parallel volume meshing face removals and hierarchical repartitioning. Comput. Methods Appl. Mech. Engrg. 174, 275-298 (1999)

5. Jurczyk, T., Głut, B.: Adaptive Control Space Structure for Anisotropic Mesh Generation. In: Proc. of ECCOMAS CFD 2006 European Conference on Computational Fluid Dynamics, Egmond aan Zee, The Netherlands (2006) 
6. Ivanov, E., Andrä, H., Kudryavtsev, A.N.: Domain decomposition approach for automatic parallel generation of 3D unstructured grids. In: Proc. of ECCOMAS CFD 2006 European Conference on Computational Fluid Dynamics, Egmond aan Zee, The Netherlands (2006)

7. Larwood, B.G., Weatherill, N.P., Hassan, O., Morgan, K.: Domain decomposition approach for parallel unstructured mesh generation. Int. J.Numer. Meth. Engng. 58, 177-188 (2003)

8. Głut, B., Jurczyk, T., Breitkopf, P., Rassineux, A., Villon, P.: Geometry Decomposition Strategies for Parallel 3D Mesh Generation. In: Proc. of Int. Conf. on Computer Methods and Systems CMS 2005, Kraków, Poland, vol. 1, pp. 443-450 (2005)

9. Jurczyk, T., Głut, B., Breitkopf, P.: Parallel 3D Mesh Generation using Geometry Decomposition. In: AIP Conf. Proc. NUMIFORM 2007, Materials Processing and Design: Modeling, Simulation and Applications, Porto, Portugal, June 17-21, vol. 908, pp. 1579-1584 (2007)

10. Ito, Y., Shih, A.M., Erukala, A.K., Soni, B.K., Chernikov, A., Chrisochoides, N.P., Nakahashi, K.: Parallel unstructured mesh generation by an advancing front method. Mathematics and Computers in Simulation 75, 200-209 (2007)

11. Sziveri, J., Seale, C.F., Topping, B.H.V.: An enhanced parallel sub-domain generation method for mesh partitioning in parallel finite element analysis. Int. J. Numer. Meth. Engng. 47, 1773-1800 (2000)

12. Jurczyk, T.: Efficient Algorithms of Automatic Discretization of Non-Trivial Three-Dimensional Geometries and its Object-Oriented Implementation. PhD thesis, AGH University of Science and Technology, Kraków, Poland (2007), http://home.agh.edu.pl/jurczyk/papers/phd-jurczyk.pdf

13. Jurczyk, T., Głut, B.: Organization of the Mesh Structure. In: Bubak, M., van Albada, G.D., Sloot, P.M.A., Dongarra, J. (eds.) ICCS 2004. LNCS, vol. 3037, pp. 646-649. Springer, Heidelberg (2004) 